\title{
ÜBER DIE TECHNISCHE SELBSTBESTIMMUNG DES MENSCHEN \\ Ein begrifflicher und phänomenologischer Beitrag zur Erklärung der Fragen nach Eugenik, Transhumanismus, und dgl. *
}

\author{
Andrea Altobrando** \\ https://orcid.org/0000-0001-6531-2361 \\ andalt@gmail.com
}

\begin{abstract}
Especially after the disasters of WWII, the relationship between human nature and technology has mainly been discussed in "Promethean" terms. That is to say, it has been discussed as it concerns the capacity, and perhaps the necessity, of humans to think and act technically, i.e., by means of technical devices, as well as to technically transform the environment. Now, as a consequence of the more recent, impressive advancements and achievements in biotechnology, pharmaceutics, etc. the new questions in this field seem more reminiscent of the story of Icarus. The enigmatic core of this myth concerns the capacity to somehow change the human nature, the need to do so, and, aside from just the risks, what the goals of such modifications would be. In this article, a simultaneously conceptual and phenomenological analysis will be carried out regarding the relationship between technical capacities, technical products, human needs, and human desires. Although such an analysis should allow one to better frame also current questions concerning the ethical sides of technical (self-)interventions into human "nature", the aim of this article
\end{abstract}

* Article submitted on 13/11/2019. Accepted on 08/04/2020.

** China University of Political Science and Law. Beijing, China. 
is just to contribute to the clarification of the link between being human and technology.

Keywords Self-determination, human nature, technology, self-modification.

ABSTRAKT Insbesondere nach den Katastrophen des Zweiten Weltkriegs wurde die Beziehung zwischen menschlicher Natur und Technologie hauptsächlich in „, Promethean “-Begriffen diskutiert. Es wurde entsprechend diskutiert, da es darum geht, die Fähigkeit und möglicherweise die Notwendigkeit des Menschen, technisch zu denken und zu handeln, d. h. mit Hilfe technischer Geräte. Dies betrifft ebenso die menschliche Möglichkeit, die Umwelt technisch zu verändern. Infolge der letzteren, beeindruckenden Fortschritte und Errungenschaften in den Bereichen Biotechnologie, Pharmazie usw., beziehen sich die neuen Fragen, die an diese Felder gestellt werden können, eher auf die Geschichte von Ikarus. Der rätselhafte Kern dieses Mythos entspricht der Fähigkeit, die menschliche Natur irgendwie zu verändern, sowie die Notwendigkeit, dies zu tun, und, abgesehen von den Risiken, die Ziele solcher Modifikationen. In diesem Artikel wird eine gleichzeitig begriffliche und phänomenologische Analyse hinsichtlich der Beziehung zwischen technischen Fähigkeiten, technischen Produkten, menschlichen Bedürfnissen und menschlichen Wünschen, durchgeführt. Obwohl eine solche Analyse es ermöglichen sollte, aktuelle Fragen ebenso zu den ethischen Seiten technischer (Selbst-) Eingriffe in die menschliche „Natur" besser zu erfassen, soll dieser Artikel nur dazu beitragen, den Zusammenhang zwischen Menschsein und Technologie zu klären.

Schlüsselwörter Selbstbestimmung, menschliche Natur, Technik, Selbstmodifizierung.

\section{Einleitung}

Die Frage nach der Beziehung zwischen Technik und menschlicher Natur ist gewiss nicht neu. Die neueren und zunehmend mächtigeren Erfindungen der Technik - vor allem in den Gebieten der Biotechnologie, Gentechnik, Pharmakologie, usw. - haben aber eine gewisse neue Dringlichkeit und Sachlichkeit dieser Frage verleiht. Nach dem II. Weltkrieg wurde die Zerstörungsund (eventuell) Überwachungskraft der Technik am meisten diskutiert. Seit den 80. Jahren des letzten Jahrhunderts wurden aber Worte und Begriffe wie Biopolitik, Posthumanismus, Anthropotechnik, Human Enhancement und derer Verwandten immer mehr ins Zentrum von akademischen sowie extra- 
akademischen Debatten gezogen. Die sich daran knüpfende Hauptfrage wird dann, ob und inwiefern der Mensch sich selbst technisch gestalten darf - oder sogar muss. Wenn wir in der Tat einer gewissen Tradition folgen, der zufolge der Mensch wesentlich mit der Technik verbunden ist, könnte man sogar behaupten, dass der Mensch sich selbst technisch gestalten muss, um Mensch zu bleiben. Wir werden im Folgenden sehen, wie das genau zu verstehen ist.

Die Frage nach der Legitimität einer technischen Modifizierung eines Menschenwesens wurde von Jürgen Habermas in seinem bekannten Aufsatz »Auf dem Weg zu einer liberalen Eugenik? Der Streit um das ethische Selbstverständnis der Gattung « $^{1}$ in Bezug auf die gentechnische Manipulation von Menschenkindern behandelt. Was Habermas bezüglich der moralischen Handlung der Eltern - und einer Menschengeneration überhaupt - gegenüber ihren (zukünftigen) Kindern sagt, sollte m.E. auch in Bezug auf die Selbst- und Heterobestimmung von Erwachsenen beachtet werden. Kurz gesagt, sollte man die genannte Frage als eine Frage nach der technischen Selbstmodifizierung des Menschen im Allgemeinen auffassen. ${ }^{2}$ Habermas unterscheidet zwischen dem ethischen Selbstverständnis des Menschen und seiner moralischen Bestimmung. Habermas Meinung nach gäbe es ein gewisses Selbstverständnis aller Menschen, das ihnen erlaubt, sich als moralische Personen zu verstehen, d.h. sich als Subjekte irgendeiner Moral zu sehen bzw. zu fühlen. Die modernste Technik - besonders die Gentechnik - erlaubt eine angeblich technische »Produktion« von Kindern, welche das erwähnte ethische Selbstverständnis potentiell gefährden würde. Man kann nicht ausschließen, dass gentechnisch hervorgebrachte Kinder keine sich ethisch selbstbestimmenden Wesen mehr wären. Und selbst wenn sie doch noch selbstbestimmende Wesen wären, könnten sie möglicherweise ihre Selbstbestimmung nicht ethisch auffassen. Das bedeutet, dass die neueste Gentechnik eine Selbstmodifizierung der Menschengattung theoretisch erlauben könnte, die die Anerkennung zwischen den Individuen als ethischen und moralischen Wesen - und auch innerhalb eines Individuums, d.h. einer Person mit sich selbst - ausschließe. Angesichts dieser Möglichkeit bzw. diesem Risiko versucht Habermas Klarheit unter einer Menge von vermischten Intuitionen zu schaffen. ${ }^{3}$

1 Vgl. Habermas (2001).

2 Ob eine genetische Modifikation eines Erwachsenen tatsächlich möglich ist, oder ob sie eher reine Sciencefiction ist, ist für die Fragestellung des vorliegenden Beitrags irrelevant, insofern es sich mit der de jure Frage nach den Möglichkeitsbedingungen einer technischen Selbstmodifizierung des Menschen und deren möglichen Konsequenzen im Allgemeinen beschäftigt.

3 Vgl. Habermas (2001), 55. 
Mit diesem Artikel möchte ich einen weiteren Beitrag einigermaßen in dieselbe Richtung von Habermas anbieten. Genauer gesagt, möchte ich die Frage nach einer technischen Selbstmodifizierung erhellen. Zu diesem Zweck werde ich sowohl begriffliche als auch phänomenologische Analysen anwenden. Wie angedeutet, werde ich aber die Sachlage szs. aus einer allgemeineren Perspektive als Habermas betrachten. Ich werde nämlich die spezifisch technische Selbstbestimmung des Menschen nicht so sehr in Bezug auf die intergenerationellen Beziehungen der Menschengattung behandeln, welche tendenziell technische Heteromodifiezierungen unter gesonderten Individuen betreffen, sondern die technische Selbstmodifizierung bzw. -gestaltung in Bezug auf eine streng genommene Modifizierung bzw. Gestaltung des Menschenwesens von sich selbst berücksichtigen, - abgesehen davon, ob es eine Selbstbestimmung der Gattung oder des Individuums ist. Der reflexive Aspekt einer solchen Handlung liegt also im Mittelpunkt meiner Überlegungen. Ich werde die Möglichkeit im Allgemeinen betrachten, dass der Mensch bzw. ein Mensch sich technisch auf eine Weise selbstbestimmt, die möglicherweise seine »Natur« ändert. Meine Absicht ist mit folgenden Punkten zu erhellen: 1. Wie die (Selbst-)Instrumentalisierung der biologischen Konstitution des Menschen prinzipiell möglich ist, und 2. ob die damit verbundenen Selbstmodifizierung des Menschen notwendig oder nur eventuell möglich ist. Dabei sollte sich auch 3. ein gewisser Beitrag zur Erklärung der Frage, wie und warum ein Individuum einer solchen Neigung bzw. einem solchen Begehren nach Selbstmodifizierung fällig sein könnte, ergeben. Ich möchte also sowohl die phänomenologischen Strukturen und Bedingungen eines sich selbst technisch und bewusst bestimmenden und modifizierenden Wesens, als auch das Gefüge seines Bedürfnisses von Selbstmodifizierung erhellen. Damit hoffe ich 4. eine klarere Einsicht sowohl in die Grenzen der technischen Selbstmodifizierungen als auch in einige möglichen Bestrebungen nach der Überwindung solcher Grenzen im Allgemeinen zu gewinnen. Zu diesem Zweck werde ich schließlich eine einigermaßen extreme Form von Selbstbestimmung betrachten, nämlich die Möglichkeit einer technischen Bestimmung der eigenen »naturwüchsigen« bzw. »gegebenen« Bedürfnisse bzw. Begierde. M.E. wird ein solches Verfahren in der Tat erlauben, sowohl die theoretische Möglichkeit einer technischen Selbstaufhebung des Menschen als solchen, als auch ihre möglichen und spezifisch technischen Selbstgestaltungen einzuschätzen.

Dieser Artikel befasst sich jedoch nur mit der allgemeinen Frage nach der Beziehung zwischen menschlicher Natur und Technik. Er befasst sich nicht direkt mit ethischen Fragen und zielt nicht darauf $a b$, einen Beitrag zur Philosophie der Technik als solcher zu leisten. Ich werde eine allgemeine, 
jedoch präzise Definition der Technik erarbeiten, die mir ermöglichen wird, Folgendes zu untersuchen: 1. wie ein Wesen im Allgemeinen sein muss, um fähig zu sein, technologisch zu denken und zu handeln; und 2. in welchem Umfang und in welchem Sinne der Mensch eben Technik braucht, um seine Identität als Mensch zu bewahren. ${ }^{4}$

Es ist doch sattsam bekannt, dass der Mensch seit der Antike durch seine »technische« Fähigkeit vom Tier zu unterschieden sei. Bevor diese Idee in der Philosophie überlegt und analysiert wurde, wurde sie schon im Mythos, bekanntlich in dem Mythos von Prometheus, ${ }^{5}$ dargestellt. Im Mythos kommt die technische Fähigkeit von den Göttern, und ist meistens mit der Idee verbunden, dass der Mensch eine Art schwaches Wesens ist, d. h ein Mängelwesen. Diese ist auch eine Idee, die später in der Philosophie aufgenommen worden ist. ${ }^{6}$ Mit dieser Auffassung des Menschen ist noch eine weitere verbunden: Der Mensch ist ein »nicht-festgestelltes Tier«, wie Nietzsche sagte. ${ }^{7}$ Wie diese Auffassung mit dem Begriff der Technik zusammenhängt, ist leicht zu sehen. Der genannten Auffassung zufolge bestehe das Unterscheidungsmerkmal des Menschen von anderen Lebewesen darin, dass er technisch handeln kann und muss. Die Technik ist das, was den Menschen aus dem Naturzustand herausführt - und im Naturzustand kann der Mensch als Mensch angeblich nicht überleben. Folglich könne der Mensch auf keine feste Natur zurückgeführt werden. In diesem Sinne kann man sagen (und ist gesagt worden), dass die »Natur« des

4 Auf der Grundlage von Mitcham (1994) besteht heute die Tendenz, zwischen „humanities philosophy of technology“ und „engineering philosophy of technology“ zu unterscheiden. Dieser Artikel könnte wahrscheinlich als der ersten Tradition zugehörig angesehen werden. Er beschäftigt sich gar nicht mit Analysen von spezifischen Technologien und technischen Handlungen. Darüber hinaus sieht es so aus, als ob man in der „analytischen Philosophie der Technik" nicht so einfach von einem allgemeinen Begriff von Technik sprechen kann; vielmehr führt man konzeptuelle Analysen darüber durch, was in verschiedenen technologischen Praktiken und insbesondere in der Arbeit von Ingenieuren geschieht. In dieser Hinsicht behaupten Franssen, Lockhorst, van de Pol (2018), dass »Humanities philosophers of technology tend to take the phenomenon of technology itself largely for granted; they treat it as a 'black box', a given, a unitary, monolithic, inescapable phenomenon «. Ich finde eine solche Charakterisierung sehr zweifelhaft, aber das ist nicht der Ort darüber zu sprechen. Ich möchte hier nur die These verteidigen, dass eine allgemeine Definition - was man phänomenologisch auch eine Wesensbeschreibung - irgendwelches Phänomens nicht unbedingt einer Reduktion desselben zu einem monolitischen und unausweichlichen entspricht. Wie auch immer, werde ich hier eine ganz allgemeine Definition von Technik erarbeiten und billigen, von der aus ich mich mit der Frage nach der technischen Selbstbestimmung des Menschen auseinandersetzen werde. Darüber hinaus möchte ich ohne Umschweife sagen, dass ich nicht einmal behaupte, dass dieser Artikel im spezifischen Bereich Philosophie der Technologie gelten soll. Er gehört vielmehr dem Gebiet der philosophischen Anthropologie.

5 Für eine philosophisch tiefgehende Auslegung des Mythos von Prometheus und seiner Bedeutung für das griechische (und im Allgemeinen westliche) Menschenbild vgl. Kerényi (1959).

6 Der klassische Bezugspunkt für die philosophische Verarbeitung des Prometheus-Mythos ist natürlich Platons Protagoras. Im 20. Jahrhundert wurde die Idee des Menschen als Mängelwesen besonders von Arnold Gehlen vertreten und ausgearbeitet, der sie aus Herders Abhandlung über den Ursprung der Sprache nahm.

7 Vgl. Nietzsche (1886), Drittes Hauptstück, §62. Auch diese Idee wurde von Gehlen wiederaufgenommen und ausgearbeitet. 
Menschen darin besteht, sich selbst immer wieder bestimmen zu müssen. ${ }^{8}$ Selbst die »übersinnliche« Bestimmung des Menschen ist vor allem (mehr oder weniger sinnvoll) denkbar, weil er in der Natur keinen festen Ort und keinen festen, präetablierten Werdegang hat.

Man kann also sagen, dass der Mensch sein eigenes Leben zumindest auch durch die Technik gestalten muss. Heißt das aber auch, dass der Mensch sich selbst durch die Technik gestalten muss? Ich sage hier absichtlich »muss « und nicht »soll« oder »darf«. Bevor man eine ethische Frage stellen kann, muss man verstehen, ob und inwiefern notwendig ist, dass der Mensch nicht nur die eigene Welt, sondern auch sich selbst durch die Technik gestaltet. Um das zu verstehen, muss man aber vorher festlegen, worin die Bedeutung von Technik besteht, und was es heißt, dass der Mensch technikfähig und technikbedürftig ist - nämlich ob und inwiefern der Mensch das »natürliche« Bedürfnis hat, (auch) sich selbst technisch zu gestalten. Auch dies Problem ist nicht neu. Man findet es spätestens im Mythos von Daidalos und Ikaros symbolisiert. Eine begriffliche und phänomenologische Erhellung des enigmatischen Kerns dieser Ikone des »Human Enhancements « wird bei den Debatten zur Biopolitik, Bioethik, Post-Humanismus, usw. behilflich sein.

Des Öfteren haben sich Philosophen darauf konzentriert, das Risiko von »Entmenschlichung" der technischen Modifizierung des Menschen hervorzuheben und zu diskutieren. ${ }^{9}$ Diese Positionen sind gewiss tendenziell konservativ, da sie irgendwelche Form von Erhaltung der menschlichen Natur anstreben. An sich wäre aber ein solcher Konservatismus wahrscheinlich vernünftig und verständlich. Er würde einer vernünftigen Besonnenheit entsprechen. Das Problem ist eher, dass diese Art von Konservatismus eine naturalistische Position bezüglich des Wesens des Menschen impliziert, die dann aber die moralischen Ansprüche zur Erhaltung des Menschenwesens, so wie er gerade ist, erheblich abschwächt. Die Transhumanisten haben es dann einfach zu zeigen, dass die Natur selbst des Menschen keiner festen Gestalt entspricht, und dass es theoretisch, oder sogar rechtlich, keine »natürlichen« Grenze zu seiner Modifizierung gesetzt werden können. ${ }^{10}$

Wie schon gesagt, möchte ich in den folgenden Paragraphen einen Beitrag zur Aufklärung der altbekannten und heutzutage aufdringlicheren Frage nach

8 Vgl. z. B. Virno (2010), der die »rekursive« und unaufhörliche Selbstbestimmung des Menschen auf seine Sprachfähigkeit zurückführt, und seine daraus folgende syntaktische Beziehung zu der eigenen Erfahrung und deren Inhalten zählt. Virno nimmt so Ideen und Theorien von Lew Semjonowitsch Wygotski und Noam Chomsky über Sprache, Denken und Erfahrung wieder auf.

9 Vgl. z.B. Mehlman (2012).

10 Vgl. z.B.: Engelhardt (1990); Bostrom (2003). 
der technischen Bestimmung des Menschen bzw. der menschlichen Natur anbieten, der sich auf sowohl begriffliche als auch phänomenologische Analysen einer technischen Handlung, eines technischen Artefaktes und eines technischen Bewusstseins stützt. Auf der Basis sowohl einiger allgemeinen Begriffsklärungen zur Sache der Technik, als auch einer »Husserlschen« systematischen Analyse der Stufen des Bewusstseins möchte ich hier vornehmlich zeigen, dass die Frage nach der technischen Gestaltung bzw. Modifizierung des Menschen zunächst und vor allem keine Frage nach der Natur des Menschen oder nach den Bedingungen eines menschlichen Lebens, sondern eine Frage nach den Bedürfnissen und Begierden sein sollte, die eine solche Gestaltung bzw. Modifizierung anfordern. Anstatt sich auf die Frage nach der Verbesserung oder Verschlechterung der menschlichen Natur zu konzentrieren, sollte man genauer bedenken, welche Gesellschaft bzw. Welt sich man sich wünscht, indem man sich mit solchen (möglichen wie auch unmöglichen) Modifizierungen auseinandersetzt. In dieser Hinsicht könnte dieser Artikel gewissermaßen als ein Beitrag innerhalb der von Habermas entworfenen Forschungs- und Fragestellungsrichtung verstanden werden. Die Frage nach der technischen Selbstbestimmung des Menschen ist zuletzt eine politische Frage und keine bloß moralische Frage. Sie betrifft in der Tat immer (auch) das Miteinandersein des Menschen - inklusiv das Mit-sich-selbst-Sein. In dieser Hinsicht können natürlich auch Fragen der Bioethik erst auf der Basis von Klärungen unserer Menschenbegriffen mittels einer Unterscheidung ihrer Identitätskriteria kritisch und rationell durchgeführt werden. Quante (2002) hat wichtige Beiträge in diese Richtung geleistet. ${ }^{11}$ Hiermit hoffe ich, durch phänomenologisch-husserlsche Analysen des Geistesbegriffes, der Beziehung zwischen verschiedenen Technikbegriffen, und deren Verhältnissen mit spezifischen Strukturen des menschlichen Geistes der weiteren Erhellung der begrifflichen Basis heutiger ethischen, bioetischen, rechtlichen und politischen Debatten über die Natur des Menschen und derer Modifizierung beizutragen.

Ich werde hier keine konkreten bzw. empirischen, weder politischen noch rechtlichen Fragen nach technischen Interventionen behandeln, sondern nur einige allgemeinen Elemente und Strukturen zu erhellen versuchen, die bei allen rationalen Überlegungen zu den technischen Interventionen auf die »menschliche Natur« berücksichtigt werden sollten. Meine Frage ist also

11 Siehe auch Quante (2010). Obwohl diese sich als eine Studie über Hegel präsentiert, zeigt Quante (2011) wie die Klärung des Geistesbegriffes fundamental ist, um Fragen der Ethik und der Bioethik sinnvoll und wirklich zu diskutieren. In der Tat könnte das ganze Buch als eine Vorbereitung zur Auseinandersetzung mit den Themen seiner zwei letzten Kapitel sehen, nämlich mit den Fragen nach personaler Autonomie und ihren Grenzen. 
nicht, ob der Mensch sich selbst technisch gestalten darf, oder soll, sondern ob er sich selbst technisch gestalten muss. M.a.W. setzte ich mich hier mit der Frage auseinander, ob der Mensch, wenn nicht individuell zumindest als Spezies, zwangsläufig die eigene »natürliche« Konstitution durch irgendwelche Technik umgestalten und modifizieren muss. Meine ist offensichtlich keine Frage nach einer »zweiten Natur«, d.h. einer geistlichen bzw. gesellschaftlichen und kulturellen Natur des menschlichen Tiers, sondern spezifischer nach einer szs. »materiellen Natur«, die durch technische Interventionen - in dem im Folgenden näher bestimmten Sinne - hervorgebracht wird. Die Hauptfrage dieses Artikels kann also auch folgendermaßen ausgedrückt werden: Ist das Wesen des Menschen unausweichlich zur technischen Selbstmodifizierung bzw. -gestaltung bestimmt, sodass jedes Widerstreben gegen ein solches Schicksal eine Art von Unterdrückung der »Urnatur« des Menschen ist? Oder wird das Streben nach der Modifizierung des Menschen jeweils von gewissen szs. empirischen und akzidentellen Faktoren verursacht, die auch gerade gegen die »Urnatur« des Menschen (als sowohl technisch als auch nicht-technisch selbstbestimmungsfähigen Wesens) gerichtet sein könnten?

Ich werde mit einigen allgemeinen Differenzierungen des Technikbegriffs anfangen, die für das Verständnis der technischen Qualifizierung eines beliebigen Wesens, einer beliebigen Handlung, und eines beliebigen Produkts notwendig sind (Par. II). Folglich werde ich die Beziehung zwischen Technik, Bedürfnissen, und Nutz problematisieren (Par. III und IV). Das Resultat dieser Überlegung wird die Feststellung sein, dass ein technisch handelndes Wesen nicht nur der Zwecke und der Mittel seiner (technischen) Handlung, sondern auch der Bedürfnisse, wozu man die technische Handlung selbst hervorbringt, bewusst sein muss. Um die Struktur eines solchen Wesens systematisch zu begreifen, werde ich dann Husserls Analysen bezüglich der verschiedenen Stufen des Bewusstseins heranziehen (Par. V). Das wird zur Erhellung führen, dass das technisch handelnde Wesen notwendigerweise ein "geistiges « Wesen ist, denn nur ein solches Wesen kann seine Handlung richtig planen, und dazu sich selbst als Teil seines Plans betrachten. Ich werde also die Beziehung zwischen geistigen Fähigkeiten und Technik weiter hinterfragen (Par. VI und VII), und damit werde ich schließlich in der Lage sein, die Frage zu betrachten, ob und wie der Mensch als technisches Wesen auch sich selbst technisch modifizieren und gestalten muss (Par. VIII). Zum Schluss werde ich hervorheben, 1. wie man die Beziehung zwischen Bedürfnissen und ihrer technischen Befriedigung auf der Basis der hervorgehenden Paragraphen verstehen sollte, und 2. dass die Frage nach der technischen Bestimmung des Menschen unausweichlich die Untersuchung der dahinterstehenden Bedürfnisse bzw. Begierde - oder Interessen - impliziert (Par. IX). 


\section{Technik als Prozess und Technik als Produkt}

Das Wort »Technik« ist (zumindest) doppeldeutig: Einerseits bedeutet es einen Prozess bzw. eine Handlung, andererseits ein »Ding «. ${ }^{12}$ Im ersten Sinne ist Technik die Hervorbringung von Produkten auf der Basis eines Designs bzw. Plans im Hinblick auf ein Ziel; im zweiten Sinne bedeutet Technik ein Artefakt, das zur Befriedigung gewisser Bedürfnisse bzw. Anforderungen dient.

Die zwei Bedeutungen sind offensichtlich miteinander verbunden: Wir können nur das als technisches Artefakt anerkennen, was auf der Basis eines Plans zu einem gewissen bzw. bewussten Zweck hervorgebracht worden ist; und eine Erzeugung ist nur dann technisch, wenn sie auf ein Produkt abzielt, das einem Zweck dient.

Ein wichtiges Merkmal der sowohl als Prozesses als auch als Produkts verstandenen Technik besteht darin, dass sie nicht nur einem Zweck dient, sondern dass dieser Zweck bewusst ist. Mit anderen Worten: Dinge und Prozesse sind nur insofern als technisch erkenntlich, wenn sie als zielgerichtet anerkannt werden. Sie sind wesentlich mit Zweckrationalität bzw. Planmäßigkeit verbunden. ${ }^{13}$ Sowohl technische Dinge als auch technische Prozesse müssen »nützlich« sein, und ihren Sinn nicht in sich selbst erschöpfen. Ein Beispiel kann hier hilfreich sein: Ein Pfirsich kann als nützlich angesehen werden, wenn er nämlich dazu dient, den Hunger zu stillen; in diesem Fall erschöpft sich aber der Nutzen des Gegenstandes in der Nutznießung desselben. Bei einem technischen Ding und bei einem technischen Prozess ist das nicht der Fall: Der technische Gegenstand bzw. die technische Handlung ist insofern technisch, als er bzw. sie für das Erreichen von etwas Anderem da ist. Die Befriedigung, die eine technische Aktivität und ein technisches Ding bei ihrer Anwendung verursachen, ist in dieser Hinsicht ein Nebenerzeugnis bzw. eine Nebenwirkung. Sie gründet also nicht im Wesen der Technik. Dieses besteht vielmehr darin, dass Technik ein zweckmäßiges Mittel ist.

Dass der Mensch technikfähig ist, bedeutet also, dass er Mittel erzeugen kann, die der Befriedigung von Bedürfnissen dienen.

12 Vgl. Bunge (1966), 329-347.

13 Vgl. dazu die klassischen Studien von Skolimowski (1966) und Simon (1969). Beide Autoren unterscheiden Wissenschaft und Technik dadurch voneinander, dass in der Wissenschaft gesagt wird, was und wie die Wirklichkeit ist (what is), während in der Technologie darüber nachgedacht wird, wie sie sein soll (what is to be). Es könnte in Frage gestellt werden, ob eine so klare Trennung gemacht werden kann, um den Phänomenen gerecht zu sein. In diesem Artikel gehe ich davon aus, dass die Sache gewiss komplizierter ist, aber man muss eine deutliche begriffliche Unterscheidung machen, um die Dynamik und die Bedingungen der Möglichkeit einer technischen Selbstbestimmung des Menschen zu erhellen. 


\section{Natürliche Bedürfnisse und technische Mittel}

Ziehen wir ein weiteres Beispiel heran. Ein Mensch hat Zahnschmerzen. Sein Bedürfnis ist, die Zahnschmerzen loszuwerden. Damit sie aufhören, muss er eine Handlung begehen. Eine Handlung ist aber im hier dargestellten Sinne nur sofern technisch, wenn sie nicht auf direkter Weise zur Entfernung führt, sondern etwas hervorbringt bzw. verursacht, das seinerseits der Entfernung dient. Man könnte nun fragen: Wenn ein Lebewesen etwas isst, das die Entfernung verursacht, begeht es einen technischen Prozess? Nein. Aus dem bisher Gesagten ist eine solche Handlung kein technisch produktiver Prozess, weil sie keinen Gegenstand auf der Basis eines Plans produziert, der dann zur Befriedigung des Bedürfnisses führt. Ein technischer Prozess erzeugt ein Zeug, das zu einer weiteren Handlung führt bzw. für eine weitere Handlung zur Verfügung steht. In diesem Rahmen könnten wir das Essen eines gewissen Dinges als technisch verstehen, nur indem wir die Befriedigung selbst als Werkzeug sehen. Und so kommen wir zu dem wahrscheinlich wesentlichsten Problem der »technischen Selbstbestimmung« des Menschen oder irgendeines Lebewesens.

Wir haben schon erwähnt, dass die Zweckhaftigkeit maßgebend ist, um etwas als technisch anzuerkennen. Die Zweckmäßigkeit ist also ein Hauptmerkmal der Technik. Der Zweck muss aber bewusst sein. Sowohl ein technischer Prozess als auch ein technisches Ding sind Mittel und kein Zweck. Insbesondere ist ein Prozess technisch, wenn er etwas hervorbringt, das einem gewissen Zweck dient, und nicht selber Zweck ist. Ein technischer Prozess ist also eine Produktion von Mitteln und nicht die Realisierung von Zwecken. Wenn wir aber eine Befriedigung als Produkt eines technischen Handelns ansehen, wird die Befriedigung ein Mittel. Die unentbehrliche Frage ist dann folgende: »ein Mittel wofür?«.

Lassen wir momentan diese Frage offen. Gehen wir zurück zur Idee des Menschen als technikfähigen und technikbedürftigen Wesens.

\section{Technik und Nutz}

Dass der Mensch technikfähig ist, bedeutet, dass er ein Wesen ist, das zielgerichtet planen kann. Eine technische Handlung ist also nicht nur eine zielgerichtete Handlung, sondern auch eine denkgeleitete Handlung. Technisch handeln bedeutet, dass man eine Einsicht in die verfügbaren Dinge, Elemente, Situationen hat, und dass diese Einsicht in der Lage ist, solche »Sachen« als nützlich für die Hervorbringung von etwas anzuerkennen, das einem gewissen Zweck dient. Das Planen geht mit dieser Einsicht zusammen: Man nimmt 
praktisch nur wahr, was man benutzen kann, um ein Artefakt zu produzieren, und nicht direkt, was Befriedigung verursacht.

Offensichtlich ist diese Sachlage nicht so einfach. Man sollte sich in der Tat fragen, wie man etwas als nützlich ansehen kann, wenn man im Voraus kein Modell von den zu erzeugenden Artefakten hat. Diese ist aber eine Frage, die wir hier nicht behandeln können, weil sie zu weit weg von unserem Hauptproblem führen würde. Sagen wir einfach, dass das technikfähige Wesen in der Lage ist, Sachen, d.h. sowohl Dinge als auch Prozesse, als Baumaterialien zu sehen.

Diese Art von »Anschauung« setzt die Fähigkeit zu denken voraus, bzw. fällt mit einem Modus des Denkens zusammen. Man muss nämlich in der Lage sein, Dinge und Sachen im Allgemeinen, also auch Ereignisse, Prozesse usw., nicht nur wahrzunehmen, sondern sie in einen Plan bzw. in ein »technologisches« Bild einzuordnen. Diese Fähigkeit, Sachen außerhalb ihres wahrnehmungsmäßigen Zusammenhangs zu sehen und sie in einen anderen einzubilden, setzt die »normale « Wahrnehmung voraus. Aber nicht nur das. Sie impliziert auch, dass man seiner Bedürfnisse bewusst ist. Und die Bedürfnisse müssen gegeben sein. ${ }^{14}$ Wäre das nicht der Fall, wäre man also der Zwecke nicht bewusst, würde man nicht in der Lage sein, technisch zu handeln.

\section{Husserls Bewusstseinsstufungen und der technikfähige Geist}

Um die Situation ein wenig $\mathrm{zu}$ erhellen und einige Hauptfaktoren herauszuheben, können einige Analysen Husserls zu den verschiedenen Bewusstseinsarten und ihren gegenseitigen Beziehungen hilfreich sein, die er u. a. im zweiten Buch der Ideen darstellt. ${ }^{15}$

Für die Zwecke dieses Beitrags ist es von Bedeutung, insbesondere drei Hauptstufen des Bewusstseins zu unterscheiden: 1) die ästhesiologische Stufe, 2) die seelische Stufe und 3) die geistige.

1. Das »bloß« ästhesiologische Bewusstsein besteht ausschließlich aus "primären Inhalten «, ${ }^{16} \mathrm{~d}$. h. aus Sinnesempfindungen und Trieben bzw. Drängen. Eine genaue Beschreibung dieser Bewusstseinsebene ist zu den Zwecken dieses Beitrags nicht notwendig. ${ }^{17}$ Es reicht hervorzuheben, dass die ersten

14 Selbst wenn wir Bedürfnisse annehmen, die »künstlich« hervorgebracht worden sind, müssen sie ein anderes Bedürfnis als »Grund « ihrer Produktion als dasjenige haben, für dessen Befriedigung es benutzt wird. In diesem Sinne sind auch die technisch produzierten Bedürfnisse für die jeweilige technische Anschauung und das entsprechende technische Planen gegeben.

15 Vgl. Husserl (1952), insbesondere §§ 18-61.

16 Vgl. Husserl (1976), 192.

17 In dieser Hinsicht könnte man sagen, dass auch dieser Beitrag technisch fortgeht: Das Ziel ist die Befriedigung des Bedürfnisses, die Beziehung zwischen menschlicher Natur und Technik zu verstehen. Alles wird zu 
und fundamentalsten Bedürfnisse des Bewusstseinslebens auf dieser Ebene liegen. Wir werden später diskutieren, ob es möglich ist, dass es Bedürfnisse gibt, die nicht hier stattfinden. Jedenfalls kann man schon bemerken, dass alle Befriedigung, wenn sie stattfindet, etwas Sinnliches sein muss. Sogar eine »intellektuelle« Befriedigung ist etwas Sinnliches. Sie ist ein »Gefühl«, wenn man so will. Dass sie intellektuell genannt wird, hängt davon ab, dass sie durch eine gedankliche Aktivität hervorgebracht wird. Es sind also die Aktivitäten oder die Ursachen, die andersartig sein können, also auf verschiedenen Ebenen des Bewusstseinsstroms liegen, aber nicht die Befriedigung: Sie ist immer eine Art von Empfindung, also sinnlich erlebt.

Unter den Empfindungen sind auch die Kinästhesen zu zählen, d. h. die Empfindungen der Bewegung des eigenen Körpers. ${ }^{18}$ Sie sind ein notwendiges Moment des lebendigen Organismus, weil Kinästhese im Grunde auch schon mit dem Moment der Reaktion auf Stimuli identifiziert werden kann. Auf dieser Stufe finden sie auf eine im Grunde fast »mechanische« Weise statt: Wenn A passiert, bewegt sich das Subjekt unmittelbar B-weise. Da wir aber von einer Bewusstseinsanalyse sprechen, sollen wir nicht an eine bloß chemische bzw. physikalische Reaktion denken. Die Reaktion ist erlebt. Diesem Erlebnis entspricht die Kinästhese. Was man aber bemerken muss, ist die Tatsache, dass sie einfach erlebt ist, aber nicht vom Subjekt im eigentlichen Sinne »gesteuert «. ${ }^{19}$ Der Bewusstseinsfluss besteht hier einfach aus einer Folge von Anregungen, Reizen, Sinnesempfindungen und Kinästhesen. Ein echtes Bewusstsein des eigenen Körpers ist aber eigentlich noch nicht da. Das passiert nur, wenn die Kinästhesen mindestens zum Teil als spontan gesteuerten erfahren werden. Auf diese Weise werden sie konstitutiv für das, was Husserl den Körper als Organ nennt, und der auch als Mittel der Subjektivität erlebt wird. ${ }^{20}$ Dafür braucht man eine gewisse Befreiung von dem unmittelbaren Reaktionsmechanismus. Und das passiert auf der nächsten, oberen Stufe des Bewusstseins, die als

2. seelische Stufe bezeichnet werden kann. Die Bewusstseinseinheit, die wir hier finden, bestimmt das Gedächtnis. Das heißt, dass ein »seelisches Subjekt« nicht einfach auf die Anregungen der Sinnlichkeit reagiert, die jeweils

diesem Zweck »gesehen«. Das Endprodukt ist die Einsicht, und sie wird instrumentell zum Zwecke der »intellektuellen« Befriedigung verstanden.

18 Vgl. Husserl (1973a), 91-93, 154-163. Vgl. dazu auch Claesges (1973), XXIIIff. Innerhalb der Grenzen dieses Beitrags kann ich den phänomenologischen Unterschied zwischen Leib und Körper nicht genau verfolgen. Generell könnte man behaupten, dass der Leib mit der ästhesiologischen Ebene übereinstimmt, und dass nur die darauffolgenden Bewusstseinsstufen eine Objektivierung des Leibes als Körpers implizieren.

19 Das hängt u. a. davon ab, dass man hier eigentlich keine Trennung zwischen Subjekt und Handlung hat, d. h., das Subjekt ist alle Erlebnisse: Es besteht aus dem, was "spontan« hervorgerufen, als auch aus dem, was »passiv« erleidet wird. In einem gewissen Sinne ist hier eigentlich alles erleidet. 
stattfinden, sondern es trägt in sich die Spuren von vergangenen Anregungen und auch von den eigenen Reaktionen darauf. ${ }^{21}$ Anders als die ästhesiologische Einheit kann das seelische Subjekt Akte vollziehen, die keine bloßen Reaktionen auf die jeweiligen Reize sind, sondern die Akte werden von einer seelischen Einheit von innerlichen Gesetzen abhängig, die sogar die zeitlichen Abläufe der Erfahrungen verzeichnen.

Das bedeutet eine Abhängigkeit des seelischen Subjekts von sich selbst. Die jetzigen Erlebnisse sind nicht nur von den jetzigen Umständen abhängig, sondern auch von vergangenen Erlebnissen. Man kann hier also eine erste Stufe der »Selbstbestimmung « des Bewusstseinsganzen finden: Auch dank der vergangenen Erfahrung wird eine jetzige Handlung bestimmt. Das setzt aber voraus, dass es die Möglichkeit gibt, spontane, d. h. »innerlich gesteuerte« Erlebnisse hervorzurufen. Diese »Selbststeuerung« ist einerseits durch die Fähigkeit ermöglicht, Anregungen und Situationen »im Zeichen« von vergangenen Erfahrungen zu sehen. Wenn A passiert, erinnert man sich, dass B folgt. Deswegen spricht Husserl auch von einem System des »wenndann«:22 Das Subjekt erlebt nicht nur Triebe und Anregungen, sondern auch eine Art von Projektion von vergangenen Erlebnisse auf die gegenwärtigen. Die Reaktion wird also auch von den Vergangenheitserlebnissen abhängig. Es entsteht immer noch keine »frei« gesteuerte kinästhetische Reaktion, aber jetzt sind die Kinästhesen von der Unmittelbarkeit einigermaßen befreit. Es entstehen folglich Habitualitäten, die eine Art von »Reserve« von Reaktionen konstituieren. Da sie nicht nur von den jeweilig gegenwärtigen Umständen abhängig sind, können diese Reaktionen auch als mögliche bzw. potentielle Aktionen betrachtet werden. Durch »innere« Motivationen können sie »freigeschaltet « werden. Das seelische Subjekt kann aber noch nicht wirklich frei entscheiden. Die Spontaneität selbst steht unter einer instinktiven Gesetzmäßigkeit, also unter primären Trieben, die letztlich entscheiden, welche kinästhetischen Abläufe stattfinden. Mit anderen Worten, das Ziel der Re-aktion wird nicht gewählt, sondern sie passiert je nachdem, welcher Trieb jeweilig stärker ist. Es gibt also noch keine bewusste Distanz zwischen Anregung und Antwort. Die Umwelt des Subjekts ist breiter als die jeweilige »gegenwärtige« Empfindungswelt, und man kann von einer »inneren Kausalität « sprechen. Um eine »freie« Selbstbestimmung zu leisten, braucht man aber noch

3. ein "geistiges" Bewusstsein. Nur hier finden wir jene Bewusstseinselemente (d. h. Erlebnisse), die eine solche Selbstbestimmung 
ermöglichen, welche gleichzeitig eine Bestimmung der eigenen Ziele ist. Diese Elemente sind:

a) ein reflexives Selbstbewusstsein, also eine Art von Bild von sich selbst;

b) ein Bewusstsein der verschiedenen möglichen Reaktionen, die man angesichts einer besonderen Situation freischalten kann;

c) ein Bewusstsein der möglichen Folge dieser Reaktion.

Demzufolge gehöre einem geistigen Subjekt auch das Bewusstsein zu, dass zumindest ein Teil von dem, was sich ereignet, von der eigenen Reaktion abhängt. Das Subjekt wird m. a. W. verantwortlich für das eigene Tun. ${ }^{23}$ Das Subjekt vollzieht jetzt nicht nur Reaktionen, sondern echte Handlungen. Diese Idee der Handlung schließt ein, dass die Aktion vom Subjekt (zumindest prinzipiell) »frei« vollzogen wird. Das Subjekt entscheidet, was passiert, d. h. welche Kinästhesen stattfinden. ${ }^{24}$ Dem Subjekt sind gleichzeitig sowohl die Folgen der kinästhetischen Abfolge als auch die Tatsache, dass die jeweils gewählten Reaktionen nicht die einzigen möglichen kinästhetischen Abläufe sind, bewusst. Ihm ist also bewusst, dass das, was passiert, auch von seiner Entscheidung bzw. von seiner Zusage zu einer oder anderer der möglichen Antworten abhängt. Es gibt also eine Distanz zwischen Anregung und Antwort, der eine Phase von Unbestimmtheit entspricht. Es ist im Grunde das Moment der Beurteilung, in dem man überlegt und eine Kette von »virtuellen« Abläufen betrachtet.

Wir lassen hier die spezifische Frage nach dem freien Willen beiseite. Es ist dann leicht zu sehen, dass das gerade Dargestellte das ist, was eine technische Handlung und ein technisches Produkt ermöglicht. Nur hier kann man etwas haben, das bewusst als Mittel zu gewissen Zwecken produziert und gesehen werden kann. Nur hier ist das eigentliche Planen möglich. Gerade daraus folgt die abgedroschene Charakterisierung des Menschen als Wesens, das in der Offenheit lebt, oder, aus einer phänomenologischen Perspektive besser gesagt, das die Offenheit erlebt. ${ }^{25}$

23 Vgl. Husserl (1952), 257.

24 Deswegen spricht Husserl an einigen Stellen von der »Resistenz « als erster Handlung, weil sie das Moment ist, wo die kausal-automatische Reizreaktionskette unterbrochen wird, und den Raum für eine "gewählte» Handlung schafft. Die Resistenz wäre in dieser Hinsicht die erste Antwort der »Freiheit» bzw. die erste »aktive» Antwort.

25 Der erste »Phänomenologe«, der die menschliche Natur durch die »Weltoffenheit» kennzeichnet, ist wahrscheinlich Max Scheler: Vgl. Scheler (1976). 


\section{Technik und Geist}

Wenn wir also die Idee verfolgen, dass der Mensch ein technikfähiges Wesen ist, müssen wir anerkennen, dass der Mensch zumindest auch ein geistiges Wesen ist. Nur so kann ein Wesen den Zusammenhang zwischen Mittel und Ziel als solchen sehen, und auf der Basis dieser Anschauung handeln, $d$. h. die eigene Aktion bestimmen. Ein nicht-geistiges Wesen könnte theoretisch in der Lage sein, Instrumente zu benutzen und einigermaßen zu erzeugen, aber ihm würde das Bewusstsein der Möglichkeit verschiedener Ziele fehlen. Diese wären einfach vorausgesetzt und, banal gesagt, nicht »reflektiert«. In diesem Fall gäbe sozusagen einen Mangel an Distanz zwischen dem triebhaften Fühlen und dem Tun.

Erst der Mensch als geistiges Wesen erweist sich also technikfähig. An sich impliziert das dennoch nicht, dass er auch technikbedürftig ist. Theoretisch wäre ein geistiges Wesen möglich, das technikfähig ist, aber die Technik nicht braucht, um zu überleben oder die eigenen Bedürfnisse zu befriedigen. Lassen wir aber beiseite, was für ein Wesen das wäre, und auch inwiefern beim Menschen die Technik bis zu einem gewissen Grad notwendig ist. Wir können uns hier mit der Feststellung begnügen, dass, der Mensch seine Bedürfnisse durch die Technik befriedigen kann, indem er technikfähig ist. Er muss aber es nicht unbedingt tun. Wenn es ein Bedürfnis gibt, das mit den vorhandenen Dingen und Umständen nicht gestillt werden kann, denkt das geistige Subjekt an eine Lösung. Es kann also etwas zu diesem Zweck erzeugen, muss es aber nicht. Es gibt m. a. W. keine unvermeidliche Not zum technischen Handeln. $\mathrm{Ob}$ das Leben des Menschen im Ganzen ohne Technik möglich wäre, können wir hier jedoch nicht erörtern. ${ }^{26}$

Wie wir schon gesehen haben, ist es wichtig hervorzuheben, dass wir von einer Handlung als technischer Handlung sprechen dürfen, nur indem sie ein Produkt zur Folge hat, das nicht direkt die Befriedigung eines Bedürfnisses ist, sondern ein Mittel zu dieser Befriedigung. Wenn ich z. B. Durst habe und zum Getränkemarkt gehe, um ein Bier zu kaufen, habe ich nicht »technisch « gehandelt. Es ist bestimmt selbstverständlich, dass ich von technischen Produkten profitiere, aber meine Handlung erzeugt an sich kein Artefakt. Man könnte vielleicht dagegen einwenden: Die ganze Reihe von Aktionen, die du ein Teil seines Wesens verloren ginge, wenn er niemals technisch handeln würde, weil er sich damit auch das technische Denken abgewöhnen würde. Darüber hinaus, wie wir noch sehen werden, ist die Technik stark mit dem symbolischen Erleben des Menschen als Mensch verbunden. Das könnte bedeuten, dass der Mensch mit der Technik auch den symbolischen Aspekt seines Daseins verlieren würde. Die Frage, ob er dann noch ein Mensch wäre, müssen wir aber offenlassen. Vgl. dazu Cassirer (2004). 
vollziehst, um eine Bierflasche in deinen Händen zu halten, ist dein Artefakt. So würde man aber zu breit die Bedeutung von Artefakt verstehen. ${ }^{27}$

Wenn wir zugeben, dass ein Mensch technikfähig ist, sagen wir, dass er zumindest teilweise dazu beiträgt, seine Bedürfnisse durch Handlungen zu befriedigen, die aber zum Ergebnis nicht die Befriedigung, sondern das Mittel zur Befriedigung haben. Wenn wir kein Ding als Endprodukt einer Handlung haben, können wir eventuell von einer »Strategie« sprechen, also von etwas, das durch Pläne und Zwecke geleistet wird. Ein Spezifikum der Technik in dem hier diskutierten Sinne ist aber die Materialität des Produktes. Wenn wir behaupten würden, dass eine Handlung selbst das technische Produkt ist, das hervorgebracht wird, um etwas zu erreichen, würden wir alles durcheinanderbringen. ${ }^{28}$ Es scheint sinnvoller zu sagen, dass Artefakte nur materielle Dinge sind. Also wenn ich z. B. ein Gefäß aus einer Kokosnuss mache, um Wasser zu sammeln, dann habe ich technisch gehandelt. Damit will ich sagen, dass eine Handlung nicht als Produkt angesehen werden kann. Auf diese Gedankenspur kommen wir auf das eigentliche »Problem « unserer Überlegungen, d. h. die technische Bestimmung des Menschen.

Und so kommen wir zur Ausgangsfrage zurück. Der Mensch als geistiges Wesen hat u. a. ein Bewusstsein von sich selbst. Dieses Bewusstsein kann mehr oder weniger klar und richtig sein, aber es ist da. ${ }^{29}$ Folglich findet der Mensch auch sich selbst unter den verschiedenen Baumaterialen, die zur Verfügung stehen, um Ziele zu erreichen. Die eigentlich eigene Handlung, also die körperliche, ist eine der Unterlagen, die benutzt werden können, um Ziele zu erreichen. Nicht nur: Der Mensch kann auch die eigenen Handlungen zu gestalten versuchen, so wie sie nach einem gewissen Plan sein sollen. Aber das

27 Die ontologische Debatte über Artefakte ist in der neueren analytischen Philosophie sehr lebendig, und es gibt keine Einstimmigkeit darüber, was ein Artefakt ist (vgl. z. B. Margolis - Laurence (2007)). Trotz der Verschiedenheit der Meinungen und Theorien scheint es mir jedenfalls, dass es keine Stimme für die Einbeziehung von Handlungen und Prozesse in die »Kataloge« der Artefakte gibt.

28 Dazu kann man bemerken, dass auch rein formal gedacht, also auch abgesehen von der Materialität des Produktes, die Mittelbarkeit der Technik auf diese Weise vergessen wird. Wenn die Handlung als Produkt gesehen wird, dann sollte die Handlung als Produkt einer anderen Handlung gesehen werden, ansonsten ist sie kein technisches Produkt, also kein Ergebnis einer reflektierten Handlung. Man kann gewiss sagen, dass man Handlungen hervorrufen kann, um damit gewisse Zwecke zu erreichen, aber der Handlungsprozess wäre in diesem Fall durch eine andere bloß »gedankliche Handlung " geplant und als Ergebnis dieser Handlung gesehen. Es wäre m.a.W. eine Programmierungshandlung. Es scheint mir also weniger irreführend, nur materielle Dinge als technisch anzuerkennen. Obwohl, wie wir sehen werden, wir einigermaßen an eine Objektivierung und technische Intervention auf psychische Sachen wie die Bedürfnisse denken könnten, sollte man möglicherweise in diesem Fall von einer Art von Materialisierung der Bedürfnisse anerkennen. Offensichtlich entsprechen dieser sehr groben Vorstellung heutzutage die Neurointerventionen und -medikamente.

29 Wie bekannt steht die sowohl epistemologische als auch ontologische Problematizität des Selbstbewusstseins heute immer noch im Zentrum verschiedener Debatten in der Philosophie des Geistes. Hier kann auf das Problem nicht eingegangen werden. 
bedeutet, dass der Mensch seine eigene Körperlichkeit und sein körperliches Verhalten zur Verfügung hat, um Ziele zu erreichen. Darüber hinaus, da der Mensch den verschiedenen Handlungsmöglichkeiten angesichts einer Situation bewusst ist, und einigermaßen über sie herrscht, kann er auch entscheiden, welches Ziel er jeweils verfolgt. Das heißt, dass der Mensch entscheiden kann, welches Bedürfnis zu befriedigen.

Aber wenn es so ist, und wenn der Mensch die eigene Konstitution, vor allem die leibkörperliche, ändern bzw. bestimmen kann, sollte man dann auch zugeben, dass der Mensch in der Lage ist, die eigenen Bedürfnisse zu erzeugen, d. h. sie technisch zu bestimmen? Die Frage, ob das der Fall ist, d.h. ob die Bedürfnisse und Triebe tatsächlich technisch erzeugt werden können, werden wir hier offenlassen. Sie betrifft im Grunde das Problem der Naturalisierbarkeit der Gefühle und der Impulse bzw. Triebe, der im Rahmen dieses Beitrags im Grunde eine Objektivierung bzw. »Verkörperlichung« des Leibes entspricht. Nehmen wir einfach an, dass der Mensch die Überzeugung bzw. den Verdacht haben kann, es zu können, und fragen wir: Zugunsten welchen Bedürfnisses würde es geschehen?

\section{Technisches Bewusstsein und Selbstobjektivierung: Von den Trieben zu den Zeichen}

Das geistige Subjekt ist eine obere Stufe des Bewusstseins. Das heißt, dass das geistige Erleben auf den zwei vorherigen Stufen fundiert ist, also auf der ästhesiologischen und auf der psychischen. ${ }^{30}$ Das geistige Bewusstsein besteht in der Tat nicht nur darin, denken zu können, sondern auch sich selbst und der eigenen Handlungsmöglichkeiten bewusst zu sein. Ohne dieses Bewusstsein, und also ohne das entsprechende Korrelat, d.h. den Leibkörper als Organ, hätte der Geist kein »Material« zu bestimmen. ${ }^{31}$ Es ist also klar, dass ohne die »unteren« Stufen ein instrumentelles Bewusstsein unmöglich wäre. Man braucht erst etwas »Gegebenes «, um es nachher als Instrument aufzufassen. Ein technikfähiges Subjekt braucht also Aisthesis, Kinesis und Psyche, um sich technisch zu betätigen.

Aber neben den »Sachen «, die durch die unteren Stufen gegeben werden, nämlich Wahrnehmungs- und Erinnerungsobjekten, hat der Geist auch ein

30 Ich beziehe mich hier auf die Theorie der Fundierung, die Husserl in der dritten Logischen Untersuchung darstellt (vgl. Husserl (1984), S. 281ff). Vgl. dazu Rota (1999).

31 Vgl., e.g., Husserl (1973b), 136: »Zum geistigen ,Menschen' gehört formal notwendig ein Leib, ein Organismus, der sein System von Wahrnehmungsorganen hat«. 
reflexives Bewusstsein von sich selbst und seinen Aktivitäten, Eigenschaften, Fähigkeiten.

Das geistige Bewusstsein hat also die »unteren« Stufen als »Basis«, aber gleichzeitig auch als intentionales Korrelat. Wenn sie tatsächlich als Korrelat erfasst werden, wenn sie also »objektiviert« werden, kann der Geist sie auch als Baumaterial erfassen. Wie schon angedeutet, wird die Selbstbestimmung hier deutlich anders, als sie in der psychischen Sphäre ist, weil das Subjekt hier »wählen« kann, welches Bedürfnis es zu befriedigen versuchen möchte. Zum Zwecke einer Befriedigung kann das Subjekt Teile von sich selbst als Instrument zur Erreichung der Befriedigung verwenden. Das Subjekt kommt aber bis zu dem Punkt, wo es sein ganzes Sein (zumindest das, von dem es explizites Bewusstsein hat) als Instrument sehen kann. Das heißt, dass es sein ganzes Sein, oder zumindest seinen ganzen Leibkörper und seine Handlungen, als funktionell zur Verfolgung eines Zwecks erfassen kann. Aber welchen Zweck?

Es ist klar, dass diese »totale« Selbstobjektivierung die ganzen Reden über den Menschen als »Instrument« Gottes bzw. eines göttlichen Designs ermöglicht. In der psychischen Sphäre werden die Triebe usw. einfach erlebt, und sie wirken, ohne als Funktionen zu einem Zweck erfasst zu werden. Indem sie aber funktionell verstanden werden, verlieren sie den ursprünglichen Sinn, d. h. sie brauchen jetzt einen Sinn, zu dem sie dienen oder dienen können. Eine Hauptalternative zu der theologischen bzw. übersinnlichen Auffassung der Triebe wäre eine (naiv teleologische) naturalistische, wo gesagt würde, dass die Triebe im Dienste des Grundsatzes der Selbsterhaltung stehen. Da die Triebe aber auch gegen das »Interesse « des Individuums sind, oder sein können (denn sie können auch zu seiner Destruktion führen), ${ }^{32}$ sollte man aber zumindest eine Gattungsteleologie annehmen, was nicht unproblematisch ist. ${ }^{33}$

Diese sind auf jeden Fall altbekannte Probleme, die spezifisch die Philosophie der Biologie und die Evolutionstheorie betreffen, und die für unsere Hauptfrage nicht wirklich wesentlich sind. Man sollte hier vielmehr sagen, dass durch die Fähigkeit des Subjektes, sein ganzes bewusstes Selbst als Gegenstand zu haben, die »Unbestimmtheit« des Menschen als Menschen hervortritt. Wenn es sich selbst zum Gegenstand hat, und es technikfähig und -denkend ist, kann das Subjekt nicht nur sich selbst als Baumaterial erfassen, sondern auch die »natürlichen« Zwecke, also die »spontane« Neigung, Triebe bzw. Appetite zu 
befriedigen, nicht mehr als »Wirkursache« oder als »Endursache«, sondern als Mittel sehen. Sie sind auch etwas Materielles, das manipuliert werden kann. ${ }^{34}$

Wenn man aber weder an eine natürliche noch an eine übersinnliche Teleologie glaubt, dann wäre es die wahrscheinlich einzige vernünftige Einsicht in diese Sachlage, dass es die Triebe selber sind, die sinnstiftend sind, in dem Sinne, dass ihre Befriedigung das Ziel jeglicher Handlung ist. Gilt das aber wirklich auch für die technische Handlung und ihre Produkte? Mit anderen Worten: Kann man sicher sein, dass die technische Handlung unbedingt die Befriedigung eines Triebes oder mehrerer Triebe anstrebt? Die Antwort muss ja sein, ansonsten könnte man nicht mehr von »technischer Handlung« sprechen, denn ohne die Befriedigung eines Bedürfnisses als Zweck ist weder eine Handlung noch ein Produkt technisch. Das eigentliche Problem ist vielmehr, dass der Mensch nicht nur auch ein symbolisches Wesen ist, sondern wesentlich eine zeichenhafte bzw. signitive Beziehung sowohl zu den Sachen und Dingen als auch zu sich selbst und der Welt im Allgemeinen hat. ${ }^{35}$ Die symbolische Seite wurde gerade im letzten Jahrhundert ausgehend von der Psychoanalyse viel diskutiert. Aber für unsere Frage ist die signitive Seite noch entscheidender, weil man signitiv auf »Dinge« abzielen kann, die es eigentlich nicht gibt und nicht geben kann. ${ }^{36}$ Denken wir an das runde Viereck: Man könnte das ganze Leben (und tatsächlich haben ganze Generationen von Mathematikern es getan) mit dem Versuch einer »Quadratur« des Vierecks verbringen, obwohl niemand so etwas jemals erfahren hat. Wo kommt also das Bedürfnis her, so was zu erreichen? Aus dem signitiven Denken, dem auch Bedeutungen vorschweben, die keine eigentliche Realisierung befähigen.

Wenn wir diese Fähigkeit, unrealisierbare bzw. unerfüllbare Bedeutungen zu denken, zusammen mit den verschiedenen Symboliken, die das Leben des

34 Gewiss sind sie keine Dinge im engeren Sinne von dreidimensionalen Körpern, aber doch sind sie etwas Sinnliches und mehr oder weniger Situierbares und, obwohl vielleicht prozessual, Verharrendes, und nicht einfach Ereignisse bzw. Geschehnisse.

35 Auf die »signitive Natur des Menschen hat Carlo Sini besonders insistiert: Vgl. Sini (1981), (1989), (1991).

36 Es geht hier offensichtlich um die sogenannten »Vorstellungen ohne Gegenstände« bzw. um die »nichtexistierenden Gegenstände«, ein Thema, das besonders in der Brentano-Schule viel diskutiert wurde, und in den letzten Jahren auch gerade in Bezug auf jene Tradition wieder aufgenommen worden ist: Vgl. u.a. Benoist (2001); Benoist - Courtine (2003). Ich vertrete hier eine eher nominalistische Theorie, die in Anknüpfung an Berkeleys Empirismus (vgl. insbesondere Berkeley (1710)) behauptet, dass man zeichenhafte Vorstellungen im Sinne von meinenden Ausdrücken aufbauen kann, denen aber kein einheitlicher Sinn bzw. keine (auch nur imaginative) Anschauung entsprechen kann. Ihre Gegenstände sind also sozusagen reine flatus vocis. Die Beziehung zwischen symbolischem Bewusstsein und Technik sollte dazu auch mit Hans Blumenbergs Theorie kritisch ergänzt werden, der zufolge der menschliche Geist immer wieder mit Mythen besessen sein wird, solange die Menschheit einer Wirklichkeit ausgesetzt ist, die ihren Wünschen widersteht. In dieser Hinsicht ist der Prometheische Geist des Menschen eine Art von Reaktion auf den »Absolutismus der Wirklichkeit«: Vgl. Blumenberg (1979). Leider geht eine adäquate Auseinandersetzung mit Blumenbergs These über die Grenzen dieses Beitrags hinaus. 
Menschen auffüllen, und gemeinsam mit der Phantasiefähigkeit betrachten, sehen wir ganz klar, dass der Mensch schon die Neigung haben kann, sich selbst zu ändern, um etwas zu erreichen, das seine »natürliche« Konstitution nicht ermöglicht. Die Bedürfnisse, deren Befriedigung eine technische Handlung als Zweck haben kann, können auch jenseits der »primären « Triebe sein. Ich kann mir vorstellen, dass es ganz schön wäre, die Sonne zu streicheln, obwohl das wahre Erlebnis wahrscheinlich nicht so erfreulich wäre. Das wäre auf jeden Fall ein Ziel, das aus Anreizen hervorgeht, die noch sinnhaft sind. Ihm liegt in der Tat immer noch eine »ästhetische« Vorstellung zugrunde, selbst wenn es sich um etwas Phantastisches handelt. Den eigentlichen Unsinn hat man, wenn man auf die Befriedigung von Bedürfnissen abzielt, die keine Erfüllung erlauben, sei es auch nur eine phantastische. Und das betrifft nicht nur intellektuelle Rätsel wie die Idee eines runden Vierecks, sondern gerade auch unsere Ausgangsfrage, nämlich ob der Mensch sich ändern muss.

\section{Das Bedürfnis zur Selbstbestimmung und die Bestimmung der Bedürfnisse}

Dass das gerade Erörterte nicht so weit von unserem Ausgangsproblem liegt, nämlich ob und zu welchem Zweck der Mensch sich selbst und vor allem und sogar seine Bedürfnisse ändern muss, ist leicht gezeigt.

Aus dem vorher Gesagten sollte klargeworden sein, dass die Fähigkeit, sich selbst bewusst zu ändern, ein Spezifikum des Menschen - und, allgemeiner, geistigen Wesens - ist. Der Mensch kann »das Ganze« anschauen, also auch die Beziehung zwischen seiner Handlung und ihren Ergebnissen. Der Mensch entscheidet in diesem Sinne (zumindest partiell) die eigene Teleologie. Er bestimmt, in welche Richtung er geht. Ob das gelingt, ist eine andere Frage. Wichtig ist, dass der Mensch sich eine technische Handlung und ein technisches Produkt erdenken kann, um die Verfolgung einer von ihm selber bestimmten Richtung zu verwirklichen. Der Mensch kann also die ganze Welt, das ganze »Vorhandene« als »Zuhandenes « verstehen. ${ }^{37}$ Aber wozu? Das kann man nicht sagen. Und es gibt einen prinzipiellen Grund, weshalb man das nicht sagen kann.

Man könnte zunächst einfach behaupten, dass es nicht einen einzigen Zweck gibt. Der Zweck wird von Mal zu Mal gewählt. Das heißt natürlich nicht, dass der Mensch den Zweck erfindet bzw. schöpft, sondern nur, dass 
er jedesmal entscheidet, welchen unter den verschiedenen zur Verfügung stehenden bzw. ihm andrängenden Bedürfnissen er befriedigt. Folglich gibt es nicht ein einziges Motiv, um das Ganze als Zuhandenes zu verstehen. Man könnte sagen, dass ein Bedürfnis jedes Mal gewählt wird, zu dessen Gunsten alles als Baumaterial verstanden wird.

Das wäre aber eine knappe Antwort. In der Tat, wenn alles als Zuhandenes verstanden wird, bleibt gar nichts übrig, das als befriedigungswürdig angesehen werden kann. Dieses ist der richtige und wesentliche Grund, warum man nicht sagen kann, zu welchem Zweck alles als Zuhandenes verstanden werden kann. Man befindet sich in dem Teufelskreis, den man im 20. Jahrhundert so oft und wiederholt erörtert hat. ${ }^{38}$ Es geht in summa um die Technisierung der Welt, der Menschen, der Natur und alles Möglichen - nämlich um die sogenannte Technokratie. ${ }^{39}$ Darüber brauchen wir hier nicht zu sprechen. Beschränken wir uns lieber auf die Frage nach der technischen Bestimmung des bloßen Menschen.

Das, was wir bis jetzt gesehen haben, zeigt ziemlich deutlich, dass keine Notwendigkeit besteht, dass der Mensch sich technisch gestaltet. Das technische Handeln erfordert ein bewusstes Subjekt, das frei entscheiden kann, was zu tun, also auch, ob in etwas technisch einzugreifen ist oder nicht. Wenn der Mensch sich technisch manipulieren will, setzt das voraus, das er ein Bedürfnis befriedigen will. Wie gesagt, das könnte davon abhängen, dass er einen symbolisch bzw. phantastisch erzeugten Traum hat, dessen Verwirklichung die eigene Konstitution nicht ermöglicht. Das bildet aber kein allzu großes Problem. Rationell gesehen, also auf eben derselben Ebene des technischen Denkens, kann man einfach bestimmen bzw. berechnen, ob das Ziel tatsächlich erreichbar ist, und durch die Einschätzung des Verhältnisses von Risiko und Nutzen ganz utilitaristisch entscheiden, ob es sich lohnt, eine technische Handlung einzurichten.

Ein schwierigeres Problem haben wir, wenn man sogar die Bedürfnisse ändern möchte..$^{40}$ Hier hat man in der Tat einen einigermaßen »konkreteren « Teufelskreis. Warum sollte man ein Bedürfnis manipulieren? Man kann sich

Vgl. z.B. Horkheimer - Adorno (1947); Ellul (1954); Anders (1956 und 1980); Heidegger (1962); Bense (1966); Illich (1975); Severino (1979).

39 Vgl. Allen (1933); Gurvvitch (1949).

40 Offensichtlich ist das der Fall nicht nur in magischen Welten, wo man z. B. Zaubertränke erzeugt, die mehr oder weniger wollüstige Liebe verursachen, sondern auch in unserer aktuellen Welt, wo man Arzneimittel produziert, die zur Beseitigung gewisser sozial oder gesetzlich unannehmbaren Dränge gerichtet sind - man denke z. B. an die »chemische Kastration« -, und theoretisch auch Genmanipulationen anstreben kann, die eventuell neue »biologischen« Instinkte produzieren. Man könnte auch trivialerweise an Medikamente denken, die das Verschwinden gewisser Bedürfnisse wie das Bedürfnis zu Rauchen bewirken. 
vorstellen, ein Bedürfnis zu beseitigen. Wenn das passiert, folgt aber eine Verdrängung, eine Sublimierung oder eine Zerstörung des Bedürfnisses. Das Bedürfnis wird nicht befriedigt und folglich darf man nicht sagen, dass jenes das Bedürfnis ist, dessen Befriedigung der Endzweck der technisch eingerichteten Handlung bzw. des technischen Produktes war. Das Verschwinden eines Bedürfnisses kann nur der Zweck eines anderen Bedürfnisses sein. Und dann müsste man verstehen, was für ein Bedürfnis das ist. Wie bekannt, kann man den Verdacht anstellen, dass es ein selbstzerstörerisches Prinzip ist, das die Bedürfnisse verstummen lässt. ${ }^{41}$ Aber diesseits solcher »psychokosmologischen« Überlegungen muss man sich einfach fragen, ob man wirklich darüber im Klaren ist, was man verfolgt, indem man ein Bedürfnis verändern will. Verfolgt man wirklich etwas »Sinnvolles« im Sinne von einer Vorstellung, die kein bloßes flatus vocis ist?

Diese Frage können wir im engen Rahmen dieses Beitrags nicht beantworten. Wir haben aber schon angedeutet, dass es Bedeutungen gibt, die objektlos sind. Also ist es theoretisch doch möglich, dass man Zwecke verfolgt, die prinzipiell unerreichbar sind, weil es das ihnen Entsprechende im Grunde nicht gibt und nicht geben kann. Auf die empiristische Weisheit stützend, sollte man sich immer fragen, ob man in der Verfolgung der Realisierung einer Situation, die zunächst nur verbal angedeutet wird, nicht auf eine Summe von Ideen abzielt, die miteinander unverträglich sind. Es ist so, als ob man sich z.B. darauf fixieren würde, ein hölzernes Eisen zu realisieren oder eine (sinnliche) Berührung mit etwas Unsinnlichen zu erreichen. Generell soll unsere Hauptfrage jedenfalls sein, welchem Bedürfnis die Manipulierung eines Bedürfnisses dient.

Darüber hinaus sollte es jetzt klar sein, dass es keine Notwendigkeit einer technischen Selbstbestimmung des Menschen gibt. Es ist gewiss - obwohl wahrscheinlich nur in Grenzen - möglich, die »menschliche Natur« technisch zu bestimmen. Gerade die Natur der Technik macht es aber unmöglich, eine solche Modifizierung für notwendig zu halten. Zumindest ist sie nicht als Selbstmodifizierung möglich. Wie gesehen setzt die technische Handlung ein geistiges Bewusstsein in der Tat voraus. Ein geistiges Bewusstsein ist u. A. dadurch charakterisiert, dass es den Kurs und die Richtung seiner Handlung wählt. Das heißt auch, dass das geistige Wesen prinzipiell immer die Möglichkeit hat, von Mal zu Mal zu entscheiden, welchem Bedürfnis bzw. Interesse nachzugehen ist. Die menschliche Natur - zumindest was seine geistige Ebene 
betrifft - scheint also ein Bedürfnis zur Selbstbestimmung einzuschließen. Dies szs. »natürliches« Bedürfnis impliziert aber keineswegs, dass man sogar die eigenen Bedürfnisse bestimmt, und noch weniger, dass sie technisch modifiziert werden. Nichtdestotrotz gibt es offensichtlich eine gewisse Neigung zum Selbstenhancement, die naiv als bloße Neigung zur Selbstverbesserung gesehen werden könnte. Die unentbehrlichen ethischen und politischen Aspekte von Enhancementshandlungen - sowie auch von transhumanistischen Idealen sind besonders in den letzten Jahrzehnten reichlich untersucht und debattiert worden. Der Beitrag der obigen Analysen zu diesen und damit verwandten Themen betrifft besonders die Beziehung zwischen Bedürfnis nach technischer Selbstbestimmung und Bestimmung der eigenen Bedürfnisse. Diese Beziehung soll jetzt im Schlusswort behandelt werden.

\section{Schlusswort: Technik und natürlichen Bedürfnisse}

Als exergo seines oben erwähnten Aufsatzes zitiert Habermas eine Behauptung von Andreas Kuhlmann:

Wenn die künftigen Eltern ein extensives Maß an Selbstbestimmung einklagen, dann wäre es doch nur recht und billig, auch dem künftigen Kind die Chance zu garantieren, ein autonomes Leben zu führen. ${ }^{42}$

Angesichts der obigen Analysen sollten wir sagen, dass alle Menschen und jeder Mensch sich fragen sollte, ob die jeweilige technische »Selbst«-Intervention ein autonomes Leben, d.h. eine selbstbestimmende Lebensweise verbürgt oder nicht, und ob man ein solches Leben will oder nicht. M.a.W. sollte man berücksichtigen, ob das artefakte Selbst die Bewusstseinskomponente und die betreffenden Zusammenhänge noch enthält, die wir mit Husserl als notwendige für ein (auch technisch) sich bewusst selbstbestimmendes Wesen anerkannt haben.

Wir haben hier gesehen, dass die technische (Selbst-)Modifizierung des Menschen kein Schicksal ist, und dass sie eigentlich auch nicht notwendig ist, um die menschliche Natur zu bewahren. Es gibt keine natürliche und unentbehrliche Not zur technischen Selbstmodifizierung bzw. -gestaltung des Menschen - umso weniger zur technischen Selbstaufhebung der »menschlichen Natur«. Aber es kann gewiss ein mehr oder weniger gefühltes Bedürfnis danach geben. Diesbezüglich sollte man immer berücksichtigen, dass die technische 
Selbstmodifizierung des Menschen eine Möglichkeit ist, die nach den jeweiligen Wünschen, Begierden, und Interessen eines oder einiger Menschen (oder sogar einer Gesellschaft) angeregt wird. An sich ist diese Bemerkung ziemlich trivial. Die hier ausgeführten Analysen können aber die Elemente, die im Spiel sind, und auch einige allgemeine Grundregeln des Spiels selbst, aufsteigen lassen.

Die technische Selbstgestaltung ist kein fundamentaler bzw. wesentlicher Trieb bzw. Instinkt des Menschen, der Gattung „Mensch“, noch der menschlichen Gesellschaft als solchen. Prinzipiell dürfte man also kein Argument für die technische Modifizierung der menschlichen Natur benutzen, das an das technische Wesen des Menschen in den oben diskutierten Sinnen appelliert: Die technikfähige und technikbedürftige Natur des Menschen bietet an sich nämlich kein Argument für die technische Modifizierung des Menschen an. Wenn man also für eine technische Selbstmodifizierung des Menschen plädiert, oder wenn man das Bedürfnis zur technischen Selbstgestaltung erfährt, soll man sich immer fragen, was für ein allgemeines, d.h. sowohl individuelles als auch gesellschaftliches Leben man damit erreichen möchte. Dabei darf man nicht für abgemacht halten, dass es ein solches Streben zum Wesen des Menschen als Menschen gehört.

Im Allgemeinen können wir also sagen, dass die technische Bestimmung des Menschen eventuell bedeuten könnte, dass der Mensch immer auch technisch handeln muss, und technische Artefakte zu seiner Selbsterhaltung und Selbstentwicklung braucht, solange er Mensch bleibt; aber das bedeutet noch nicht, dass er die technische Modifizierung seines Selbst benötigt, um »Mensch« zu bleiben, oder einem inneren "naturwüchsigen Drang« zur Selbstaufhebung treu zu sein. Keine technische Selbstmodifizierung ist unvermeidlich - weder normativ noch deskriptiv -, um die »menschliche Natur « als solche zu bewahren. Da aber ein technikfähiges Wesen notwendigerweise auch reflexiv selbstbewusst ist, und folglich auch sich selbst - zumindest partiell - unter seinen eigenen Objekten hat, ist es selbstverständlich, dass er auch sich selbst als Baumaterial eines technischen Verfahrens und zum Aufbau eines Artefaktes sehen kann. Nur in dieser Hinsicht ist die Möglichkeit, aber gar nicht die Not, einer technischen Selbstgestaltung des Menschen notwendigerweise immer da, solange der Mensch Mensch ist. Die technische Selbstgestaltung ist eine wesenhafte Möglichkeit des Menschen - vielleicht aber nicht eines Post-Menschen. Es gibt in diesem Sinne kein technisches Schicksal der menschlichen Selbstgestaltung, aber doch eine Grenze seiner Modifizierung, solange man noch gerade seine Möglichkeit und Macht zur echt technischen Selbstbestimmung bewahren will. Anders gesagt, es gehört zum menschlichen Sein die Möglichkeit einer Selbstaufhebung zu verwirklichen, 
der einer Entmächtigung statt einer Ermächtigung entspricht, da man gerade die Fähigkeit zur bewussten technischen Selbstmodifizierung verlieren könnte. Man sollte dann fragen, wieso man so was anstreben könnte. Wenn man genauer ein solches Szenarium bedenkt, kann man sogar eine mögliche Neigung zur Verantwortungsflucht vermuten.

Die Art und Weise der eventuellen Aktualisierung einer technischen Selbstgestaltung hängt von den jeweiligen Begierden, Wünschen und Interessen $\mathrm{ab}$, die man zu befriedigen entscheidet. Die Selbstgestaltung ist eine Macht des Menschen, d.h. sie ist Teil dessen, was der Mensch prinzipiell kann, aber nicht von dem, was er unbedingt muss. Im Gegenteil impliziert die Macht zur Selbstmodifizierung die Macht der Resistenz gegen jede - und eventuell alle - Selbstmodifizierung. Die obigen begrifflichen und phänomenologischen Überlegungen zeigen also, dass die Fragen nach der technischen Selbstgestaltung des Menschen keine bloße anthropologischen, existenziellen oder psychologisch-psychoanalytischen Fragen sind. Sie sind auch keine bloß moralischen Fragen, sondern sie konstituieren eine gesellschaftliche und vor allem politische »Sache« - Habermas würde vielleicht eine ethische sagen. Die Bestimmung des menschlichen Seins durch Technik ist eine Bestimmung der Welt, die man sich als Mensch - aber eigentlich nicht unbedingt für die Menschen bzw. für alle Menschen - wünscht.

In Bezug auf die Fragen nach der gentechnischen Bestimmung von zukünftigen Kindern schreibt Habermas: »Wie wir mit menschlichem Leben vor der Geburt (oder mit Menschen nach ihrem Tode) umgehen, berührt unser Selbstverständnis als Gattungswesen. Und mit diesem gattungsethischen Selbstverständnis sind die Vorstellungen von uns als moralischer Personen eng verwoben «. ${ }^{43}$ Was Habermas hier sagt, ist überhaupt für die allgemeine Frage nach der technischen Selbstbestimmung und -modifizierung auch eines erwachsenen Menschen gültig. Die technische Intervention auf seine eigene »Natur« entspricht in der Tat der Wahl der Person, die man sein will, und das impliziert auch ihre Offenheit oder Verschlossenheit gegenüber den Anderen - d.h. sie betrifft sein Mitsein und sein Für-andere-sein. Die Vorstellung von sich selbst, die man durch die Selbstmodifizierung bzw. -Gestaltung anstrebt, ist letzten Endes mit der Vorstellung der Beziehungen oder NichtBeziehungen verwoben, die man sich mit anderen Subjekten - sich selbst als objektiviertem Selbst eingeschlossen - wünscht. ${ }^{44}$ Es ist in der Tat klar

44 Die Frage nach der technischen (Selbst-)Bestimmung des Menschen ist evidenterweise auch mit der Frage nach seiner Ent- und Verfremdung verbunden. Neben den Untersuchungen über Entfremdung in der 
ersichtlich, dass viele technischen Modifizierungen des Menschen - vor allem im Bereich der Neuropharmakologie - auch eine Modifizierung seiner »naturwüchsigen« Bedürfnisse betrifft. Diesbezüglich sollte man dann fragen, ob solche Bedürfnisse als Beschränkung der menschlichen Freiheit oder als schädlich für den Menschen selbst, oder für irgendeine menschliche, natürliche, oder göttliche Weltgesundheit gesehen werden sollen. Es besteht nämlich die Möglichkeit, dass die Entfernung gewisser Bedürfnisse einem Drang zur Überwindung der menschlichen Grenzen als solchen entspricht.

Die Moral der Geschichte, die die obigen Analysen und Überlegungen darstellen, könnte also wie folgt zusammengefasst werden: Wenn man technische (Selbst-)Modifizierungen des Menschen abwägt, sollte man immer (zumindest auch) die Wünsche und die Bedürfnisse erwägen, die man damit befriedigen bzw. erfüllen will. Man muss vor allem sich immer darüber klar sein, dass ein Bedürfnis grundsätzlich nie durch seine technische Modifizierung befriedigt werden kann, denn die Befriedigung eines Bedürfnisses ist gerade Zweck und nicht Mittel einer Technik. Alles, was als Teil des Endproduktes einer technischen Handlung gesehen wird, kann mit dem Zweck weder der Handlung noch des Artefaktes gleichgesetzt werden. Wenn jemand also behaupten würde, dass die Befriedigung eines Bedürfnisses das Produkt seiner technischen Handlung ist, würde er implizit auch sagen, dass er das Bedürfnis selbst als Baumaterial seines Schaffens benutzt. ${ }^{45}$ Der Zweck ist also nicht wirklich die Befriedigung jenes Bedürfnisses, sondern die Modifizierung bzw. die Auslöschung des Bedürfnisses selbst. Wenn jemand aber denkt, dass man durch die Modifizierung bzw. Beseitigung eines Bedürfnisses Befriedigung erreichen kann, muss man immer fragen, die Befriedigung welchen anderen Bedürfnisses man dabei verfolget.

marxistischen Tradition sollte man hier vor allem die phänomenologischen Untersuchungen von Bernhard Waldenfels erwähnen: Vgl. insbesondere Waldenfels (2002). Innerhalb der Grenzen dieses Beitrags kann man nur die Frage aufwerfen, aber nicht beantworten, ob und wie die unausweichliche Verfremdung und Entfremdung des Menschen ein notwendiges Bedürfnis zur technischen Selbstmodifizierung impliziert, und ob die Begierde nach einer solchen Modifizierung eine Offenheit oder eher eine Verschlossenheit angesichts des Fremden darstellt. Ebenfalls wäre die neulich wieder aufgenommene und diskutierte Hegelsche Idee des Geistes als »zweiter Natur « (vgl. Menke 2013) in diesen Problemrahmen einzubeziehen. Man kann das hier aus Raumbegrenzungen nicht tun. Es sollte reichen herauszuheben, dass die technische Modifizierung des Menschen die Bedingungen einer zweiten Natur erhalten und ermöglichen muss, wenn man noch vom Menschen sprechen will. Das impliziert nach unseren obigen Überlegungen u. A. die Fähigkeit zur Selbstbestimmung. Konzeptuell würde der Transhumanismus in dieser Hinsicht nicht unbedingt ein solches Erfordernis erfüllen, d.h. eine Transhumanistin könnte theoretisch eine Überwindung des Menschen als Doppelwesen durchweg beanspruchen. Wenn eine Transhumanistin das nicht tut, dann beansprucht sie nicht wirklich ein jenseits des Menschen, sondern nur eines Teils desselben.

45 Man könnte einen solchen Fall so ausdrücken: Wenn ich so und so mein Bedürfnis $X$ modifiziere, werde ich $\mathrm{Y}$ erreichen. Die Erreichung von $\mathrm{Y}$ ist also die Endursache von der technischen Arbeit an $\mathrm{X}$. 
Wenn man also sich selbst oder einen Teil von sich selbst als Baumaterial (s)einer technischen Handlung fasst, soll man fragen, wozu der Bauprozess selbst verfolgt wird, wozu das artefakte Selbst dient, und welche Art von Mitsein - oder eventuell von »unmitseinlichem « Selbst - das erlaubt. Da wir berechtigterweise sagen dürfen, dass es keine naturhafte Not einer jeglichen »technischen Selbstmodifizierung des Menschen« gibt, und diese immer der »freien « Entscheidung des Menschen selbst als reflexiv selbstbewussten Wesens gelassen ist - oder werden soll -, können wir jetzt vielleicht auch deutlicher begreifen, dass jede Frage nach einer jeweiligen willentlichen Modifikation des Menschen eine Frage ist, die mit der politischen Natur des Menschen zu tun hat, und d.h. eine Frage nach der Art seines Zusammenseins. ${ }^{46}$

\section{Literatur}

ALLEN, R. „What is technocracy?“. New York, 1933.

ANDERS, G. „Die Antiquiertheit des Menschen. Band I: Über die Seele im Zeitalter der zweiten industriellen Revolution“. München, 1956.

. „Die Antiquiertheit des Menschen. Band II: Über die Zerstörung des Lebens im Zeitalter der dritten industriellen Revolution“. München, 1980.

BENOIST, J. „Représentations sans objet. Aux origines de la Phénoménologie et de la philosophie analytique“. Paris, 2001.

BENOIST, J., COURTINE, J.-F. (Hg.). „Husserl. La représentation vide“. Paris, 2003. BENSE, M. „Ungehorsam der Ideen. Abschließender Traktat über Intelligenz und technische Welt"“. Köln, 1966.

BERKELEY, G. „A Treatise Concerning the Principles of Human Knowledge“. 1710. BLUMENBERG, H. „Arbeit am Mythos“. Frankfurt a/M., 1979.

BOSTROM, N. „Human Genetic Enhancements: A Transhumanist Perspective“. The Journal of Value Inquiry, Vol. 37, Nr. 4, pp. 493-506, 2003.

BUNGE, M. „Technology as applied science“. Technology and Culture, Nr. 7, pp. 329-347, 1966.

CASSIRER, E. „An Essay on Man“. New Haven, 1944. . „Form und Technik“. In: . Gesammelte Werke. Bd. 17: Aufsätze und kleine Schriften (1927-1931), hg. v. B. Recki, Hamburg, 2004. pp. 139-183.

Die erste Anregung zur Verfassung dieses Beitrags kam durch die freundliche Einladung von Benedetta Bisol und Christoph Asmuth zur Tagung "Translating Doping“, die an Villa Vigoni in 2011 stattfand. Bei Benedetta und Christoph möchte ich mich hier ganz herzlich bedanken. In den folgenden Jahren hatte ich das Glück, weitere Diskussionen über das Thema mit Alice Pugliese, Haojun Zhang und Nicola Liberati führen zu dürfen. Auch innen möchte ich meinen Dank für die vielen Hinweise und Ratschläge ausdrücken. Zwei anonymen Gutachtern bin ich für viele hilfreichen Anregungen auch dankbar. Endlich möchte ich mich bei der Redaktion von Kriterion und Thomas Doyon für die sorgfältige sprachliche Korrektur herzlich bedanken. Die Endverfassung dieses Beitrags wurde dank der Unterstützung vom National Social Science Fund of China (Grant No. 17BZX085) ermöglicht. 
CLAESGES, U. „Einleitung des Herausgebers“. 1973. In: Husserl (1973a), XIII-XXVIII. DAWKINS, R. „The Blind Watchmaker“. New York, 1986.

ELLUL, J. „La technique ou 1'enjeu du siècle“. Paris, 1954.

ENGELHARDT, H. T. „Human Nature Technologically Revisited“. Social Philosophy and Policy, Vol. 8, Nr. 1, pp. 180-191, 1990.

FRANSSEN, M., LOKHORST, G.-J., VAN DE POEL, I. „Philosophy of Technology“. The Stanford Encyclopedia of Philosophy (Fall 2018 Edition), Edward N. Zalta (ed.). Available at: https://plato.stanford.edu/archives/fall2018/entries/technology/.

FREUD, S. „Jenseits des Lustprinzips“. Leipzig, 1920.

GURVITCH, G. (Hg.). „Industrialisation et technocratie“. Paris, 1949.

HABERMAS, J. „Auf dem Weg zu einer liberalen Eugenik? Der Streit um das ethische Selbstverständnis der Gattung“. In: . Die Zukunft der menschlichen Natur. Auf dem Weg zu einer liberalen Eugenik?. Frankfurt a./M., 2001.

HEIDEGGER, M. „Die Technik und die Kehre“. Pfullingen, 1962.

. „Sein und Zeit“. Tübingen, 1927.

HORKHEIMER, M., ADORNO, T. W. „Dialektik der Aufklärung“. Amsterdam, 1947. HUSSERL, E. „Ding und Raum. Vorlesungen 1907“. Husserliana XVI, hg. v. U. Claesges, Den Haag, 1973a.

. „Ideen zu einer reinen Phänomenologie und phänomenologischen Philosophie. Zweites Buch: Phänomenologische Untersuchungen zur Konstitution“. Husserliana $I V$, hg. v. M. Biemel, Den Haag, 1952.

. „Ideen zu einer reinen Phänomenologie und phänomenologischen Philosophie. Erstes Buch: Allgemeine Einführung in die reine Phänomenologie“. Husserliana III/1, hg. v. K. Schuhmann, Den Haag, 1976.

. „Logische Untersuchungen. Zweiter Band, Erster Teil: Untersuchungen zur Phänomenologie und Theorie der Erkenntnis“. Husserliana XIX/1, hg. v. U. Panzer, Den Haag, 1984.

. ,Zur Phänomenologie der Intersubjektivität. Texte aus dem Nachlass. Zweiter Teil: 1921-1928“. Husserliana XIV, hg. v. I. Kern, Den Haag, $1973 b$.

ILLICH, I. „Selbstbegrenzung. Eine politische Kritik der Technik“. Reinbek, 1975. KERÉNYI, K. „Prometheus“. Hamburg, 1959.

KUHLMANN, A. „Politik des Lebens - Politik des Sterbens. Biomedizin in der liberalen Demokratie“. Berlin, 2001.

MARGOLIS, E., LAURENCE, S. (Hg.). „Creations of the Mind. Theories of Artifacts and Their Representation“. Oxford/New York, 2007.

MATURANA, H. R., VARELA, F. J. „The Tree of Knowledge. The biological Roots of Human Understanding“. Boston, 1992.

MEHLMAN, M. J. „Transhumanist Dreams and Dystopian Nightmares“. Baltimore, 2012.

MENKE, C. „Hegel's Theory of Second Nature“. Symposium: Canadian Journal of Continental Philosophy, Vol. 17. Nr. 1, pp. 31-49, 2013. 
MITCHAM, C. „Thinking Through Technology: The Path Between Engineering and Philosophy“. Chicago, 1994.

MONOD, J. „Le Hasard et la Nécessité: Essai sur la philosophie naturelle de la biologie modern". Paris, 1970.

NIETZSCHE, F. (1886). „Jenseits von Gut und Böse“. München, 1988.

QUANTE, M. „Personales Leben und menschlicher Tod“. Frankfurt am Main, 2002.

ROTA, G.-C. „Fundierung as a Logical Concept“. The Monist 72/1., pp. 70-77, 1999.

SCHELER, M. „Die Stellung des Menschen im Kosmos“. In: . Gesammelte

Werke. Bd. IX: Späte Schriften. Bern/München, 1976.

SEVERINO, Em. „Techne. Le radici della violenza“. Mailand, 1979.

SIMON, H. „The sciences of the artificial“. Cambridge (Massachusetts)/London, 1969. SINI, C. „I segni dell'anima““. Rom-Bari, 1989. (dt. Die Zeichen der Seele. Würzburg, 1995).

. „Il simbolo e l'uomo“. Mailand, 1991.

. „Passare il segno. Semiotica, cosmologia, tecnica“. Mailand, 1981.

SKOLIMOWSKI, H. „The structure of thinking in technology“. Technology and Culture, 7, pp. 371-383, 1966.

VIRNO, P. „E così via, all'infinito. Logica e antropologia“. Turin, 2010.

WALDENFELS, B. „Bruchlinien der Erfahrung“. Frankfurt a/M., 2002. 
